\title{
A SPECIAL CLASS OF UNIVALENT FUNCTIONS WITH NEGATIVE COEFFICIENTS
}

\author{
M. K. AOUF
}

\begin{abstract}
There are many special classes of univalent functions in the unit disc $U$. In this paper, we consider the special class $P^{*}(A, B, \alpha, \beta),-1 \leq B<A \leq 1$, $-1 \leq B \leq 0,0 \leq \alpha<1$ and $0<\beta \leq 1$, of univalent functions in the unit disc $U$. And it is the purpose of this paper to show some properties of this class.
\end{abstract}

\section{Introduction}

Let $T$ denote the class of functions of the form

$$
f(z)=a_{1} z-\sum_{n=2}^{\infty} a_{n} z^{n}\left(a_{1}>0 ; a_{n} \geq 0\right)
$$

which are analytic and univalent in the unit disc $U=\{z:|z|<1\}$. Then a function $f(z)$ of $T$ is said to be starlike of order $\alpha$ and type $\beta$ if and only if

$$
\left|\frac{\frac{z f^{\prime}(z)}{f(z)}-1}{\frac{z f^{\prime}(z)}{f(z)}+1-2 \alpha}\right|<\beta \quad(z \in U)
$$

for $\alpha(0 \leq \alpha<1)$ and $\beta(0<\beta \leq 1)$. We denote by $T(\alpha, \beta)$ the class of all starlike functions of order $\alpha$ and type $\beta$. Further a function $f(z)$ of $T$ is said to be convex of order $\alpha$ and type $\beta$ if and only if $z f^{\prime}(z) \in T(\alpha, \beta)$. We denote by $C(\alpha, \beta)$ the class of all convex functions of order $\alpha$ and type $\beta$.

The classes $T(\alpha, \beta)$ and $C(\alpha, \beta)$ were studied by Gupta and Ahmad [2], Owa [7,8] and by Sekine, Owa and Nishimoto [10]. In particular, for $a_{1}=1$, these classes were studied by Gupta and Jain [3,4] and Owa [6].

Received September 3, 1993; revised September 30, 1994.

1991 Mathematics Subject Classification. Primary 30C45.

Key words and phrases. Analytic functions, univalent functions, distortion theorem. 
In this paper we introduce the class $P^{*}(A, B, \alpha, \beta),-1 \leq B<A \leq 1,-1 \leq B \leq 0$, $0 \leq \alpha<1$ and $0<\beta \leq 1$, being defined as follows:

Definition 1. A function $f(z) \in T$ is in the class $P^{*}(A, B, \alpha, \beta),-1 \leq B<A \leq 1$, $-1 \leq B \leq 0,0 \leq \alpha<1$, and $0<\beta \leq 1$, if and only if

$$
\left|\frac{f^{\prime}(z)-a_{1}}{[B+(A-B)(1-\alpha)] a_{1}-B f^{\prime}(z)}\right|<\beta \quad(z \in U) .
$$

Remark 1. (i) For $a_{1}=A=1$ and $B=-1$, the class $P^{*}(1,-1, \alpha, \beta)=P^{*}(\alpha, \beta)$ was studied by Gupta and Jain [4].

(ii) For $a_{1}=A=1$ and $B=-\mu(0 \leq \mu \leq 1)$, the class $P^{*}(1,-\mu, \alpha, \beta)=P^{*}(\alpha, \beta, \mu)$ was studied by Owa and Aouf [9].

(iii) Two subclasses $T(A, B, \alpha, \beta)$ and $C(A, B, \alpha, \beta)$ of $T$, obtained by taking $a_{1}=1$ and replacing $f^{\prime}(z)$ with $\frac{z f^{\prime}(z)}{f(z)}$ and $1+\frac{z f^{\prime \prime}(z)}{f^{\prime}(z)}$, respectively in (1.3), have been studied by the author in [1].

\section{Coefficient Estimates}

Theorem 1. Let the function $f(z)$ be defined by (1.1). The $f(z)$ is in the class $P^{*}(A, B, \alpha, \beta)$ if and only if

$$
\sum_{n=2}^{\infty} n(1-\beta B) a_{n} \leq(A-B) \beta(1-\alpha) a_{1} .
$$

The result is sharp.

Proof. Let $|z|=1$. Then

$$
\begin{array}{r}
\left|f^{\prime}(z)-a_{1}\right|-\beta\left|[B+(A-B)(1-\alpha)] a_{1}-B f^{\prime}(z)\right| \\
=\left|-\sum_{n=2}^{\infty} n a_{n} z^{n-1}\right|-\beta\left|(A-B)(1-\alpha) a_{1}+B \sum_{n=2}^{\infty} n a_{n} z^{n-1}\right| \\
\leq \sum_{n=2}^{\infty} n(1-\beta B) a_{n}-(A-B) \beta(1-\alpha) a_{1}, \text { since } B \leq 0 \\
\leq 0, \text { by hypothesis. }
\end{array}
$$

Hence, by maximum modulus principle, $f \in P^{*}(A, B, \alpha, \beta)$.

For the converse, assume that

$$
\left|\frac{f^{\prime}(z)-a_{1}}{[B+(A-B)(1-\alpha)] a_{1}-B f^{\prime}(z)}\right|=\left|\frac{-\sum_{n=2}^{\infty} n a_{n} z^{n-1}}{(A-B)(1-\alpha) a_{1}+B \sum_{n=2}^{\infty} n a_{n} z^{n-1}}\right|<\beta
$$


for $z \in U$. Since $|\operatorname{Re}(z)| \leq|z|$ for all $z$, we have

$$
\operatorname{Re}\left\{\frac{\sum_{n=2}^{\infty} n a_{n} z^{n-1}}{(A-B)(1-\alpha) a_{1}+B \sum_{n=2}^{\infty} n a_{n} z^{n-1}}\right\}<\beta .
$$

Choose values of $z$ on the real axis so that $f^{\prime}(z)$ is real. Upon clearing the denominator in (2.2) and letting $z \rightarrow 1^{-}$through real values, we obtain

$$
\sum_{n=2}^{\infty} n a_{n} \leq(A-B) \beta(1-\alpha) a_{1}+\beta B \sum_{n=2}^{\infty} n a_{n} .
$$

This completes the proof of Theorem 1:

Finally, we can see that the equality in (2.1) is attained for the function

$$
f(z)=a_{1} z-\frac{(A-B) \beta(1-\alpha) a_{1}}{n(1-\beta B)} z^{n} \quad(n \geq 2) .
$$

Corollary 1. let the function $f(z)$ difined by (1.1) be in the class $P^{*}(A, B, \alpha, \beta)$. Then we have

$$
a_{n} \leq \frac{(A-B) \beta(1-\alpha) a_{1}}{n(1-\beta B)} \quad(n \geq 2) .
$$

The equality in (2.4) is attained for the function $f(z)$ given by (2.3).

\section{Distortion Theorem}

Theorem 2. Let the function $f(z)$ defined by (1.1) be in the class $P^{*}(A, B, \alpha, \beta)$. Then we have

$$
a_{1}|z|-\frac{(A-B) \beta(1-\alpha) a_{1}}{2(1-\beta B)}|z|^{2} \leq|f(z)| \leq a_{1}|z|+\frac{(A-B) \beta(1-\alpha) a_{1}}{2(1-\beta B)}|z|^{2}
$$

and

$$
a_{1}-\frac{(A-B) \beta(1-\alpha) a_{1}}{(1-\beta B)}|z| \leq\left|f^{\prime}(z)\right| \leq a_{1}+\frac{(A-B) \beta(1-\alpha) a_{1}}{(1-\beta B)}|z|
$$

for $z \in U$. The equalities hold for the function

$$
f(z)=a_{1} z-\frac{(A-B) \beta(1-\alpha) a_{1}}{2(1-\beta B)} z^{2} .
$$


Proof. In view of Theorem 1, we have

$$
2(1-\beta B) \sum_{n=2}^{\infty} a_{n} \leq \sum_{n=2}^{\infty} n(1-\beta B) a_{n} \leq(A-B) \beta(1-\alpha) a_{1},
$$

which gives

$$
\sum_{n=2}^{\infty} a_{n} \leq \frac{(A-B) \beta(1-\alpha) a_{1}}{2(1-\beta B)}
$$

Consequently, we have

$$
|f(z)| \geq a_{1}|z|-|z|^{2} \sum_{n=2}^{\infty} a_{n} \geq a_{1}|z|-\frac{(A-B) \beta(1-\alpha) a_{1}}{2(1-\beta B)}|z|^{2} .
$$

and

$$
|f(z)| \leq a_{1}|z|+|z|^{2} \sum_{n=2}^{\infty} a_{n} \leq a_{1}|z|+\frac{(A-B) \beta(1-\alpha) a_{1}}{2(1-\beta B)}|z|^{2} .
$$

Furthermore, from Theorem 1, we have

$$
\sum_{n=2}^{\infty} n a_{n} \leq \frac{(A-B) \beta(1-\alpha) a_{1}}{(1-\beta B)}
$$

Hence we have

$$
\left|f^{\prime}(z)\right| \geq a_{1}-|z| \sum_{n=2}^{\infty} n a_{n} \geq a_{1}-\frac{(A-B) \beta(1-\alpha) a_{1}}{(1-\beta B)}|z|
$$

and

$$
\left|f^{\prime}(z)\right| \leq a_{1}+|z| \sum_{n=2}^{\infty} n a_{n} \leq a_{1}+\frac{(A-B) \beta(1-\alpha) a_{1}}{(1-\beta B)}|z| .
$$

This completes the proof of Theorem 2 .

Corollary 2. Let the function $f(z)$ defined by (1.1) be in the class $P^{*}(A, B, \alpha, \beta)$. Then the unit disc $U$ is mapped by $f(z)$ onto a domain that contains the disc

$$
|w|<\frac{2(1-\beta B)-(A-B) \beta(1-\alpha)}{2(1-\beta B)} a_{1} .
$$

The result is sharp with the extremal function $f(z)$ given by (3.3).

\section{Modified Hadamard Products}

Let the functions $f_{i}(z)(i=1, \ldots, m)$ be defined by 


$$
f_{i}(z)=a_{1, i} z-\sum_{n=2}^{\infty} a_{n, i} z^{n}\left(a_{1, i}>0 ; a_{n, i} \geq 0\right) .
$$

The modified Hadamard product of $f_{1}(z)$ and $f_{2}(z)$ is defined by

$$
f_{1} * f_{2}(z)=a_{1,1} a_{1,2} z-\sum_{n=2}^{\infty} a_{n, 1} a_{n, 2} z^{n} .
$$

Theorem 3. Let the functions $f_{i}(z)(i=1,2)$ defined by $(4.1)$ be in the class $P^{*}(A, B, \alpha, \beta)$. Then $f_{1} * f_{2}(z)$ belongs to the class $P^{*}(A, B, \delta(A, B, \alpha, \beta), \beta)$, where

$$
\delta(A, B, \alpha, \beta)=1-\frac{(A-B) \beta(1-\alpha)^{2}}{2(1-\beta B)} .
$$

The result is sharp.

Proof. Employing the technique used earlier by Schild and Silverman [11], we need to find the largest $\delta=\delta(A, B, \alpha, \beta)$ such that

$$
\sum_{n=2}^{\infty} \frac{n(1-\beta B)}{(A-B) \beta(1-\delta) a_{1,1} a_{1,2}} a_{n, 1} a_{n, 2} \leq 1 .
$$

Since from Theorem 1

$$
\sum_{n=2}^{\infty} \frac{n(1-\beta B)}{(A-B) \beta(1-\alpha) a_{1,1}} a_{n, 1} \leq 1
$$

and

$$
\sum_{n=2}^{\infty} \frac{n(1-\beta B)}{(A-B) \beta(1-\alpha) a_{1,2}} a_{n, 2} \leq 1,
$$

by the Cauchy-Schwarz inequality we have

$$
\sum_{n=2}^{\infty} \frac{n(1-\beta B)}{(A-B) \beta(1-\alpha) \sqrt{a_{1,1} a_{1,2}}} \sqrt{a_{n, 1} a_{n, 2}} \leq 1 .
$$

Thus it is sufficient to show that

$$
\begin{aligned}
& \frac{n(1-\beta B)}{(A-B) \beta(1-\delta) a_{1,1} a_{1,2}} a_{n, 1} a_{n, 2} \\
\leq & \frac{n(1-\beta B)}{(A-B) \beta(1-\alpha) \sqrt{a_{1,1} a_{1,2}}} \sqrt{a_{1,1} a_{1,2}} \quad(n \geq 2),
\end{aligned}
$$

that is, that

$$
\sqrt{a_{n, 1} a_{n, 2}} \leq \frac{(1-\delta)}{(1-\alpha)} \sqrt{a_{1,1} a_{1,2}}
$$


Note that from Corollary 1

$$
\sqrt{a_{n, 1} a_{n, 2}} \leq \frac{(A-B) \beta(1-\alpha) \sqrt{a_{1,1} a_{1,2}}}{n(1-\beta B)}(n \geq 2) .
$$

Consequently, we need only to prove that

$$
\frac{(A-B) \beta(1-\alpha)}{n(1-\beta B)} \leq \frac{(1-\delta)}{(1-\alpha)} \quad(n \geq 2),
$$

or, equivalently, that

$$
\delta \leq 1-\frac{(A-B) \beta(1-\alpha)^{2}}{n(1-\beta B)} \quad(n \geq 2) .
$$

Since

$$
D(n)=1-\frac{(A-B) \beta(1-\alpha)^{2}}{n(1-\beta B)} \quad(n \geq 2) .
$$

is an increasing function of $n(n \geq 2)$, letting $n=2$ in (4.13), we obtain

$$
\delta \leq D(2)=1-\frac{(A-B) \beta(1-\alpha)^{2}}{2(1-\beta B)},
$$

which completes the proof of Theorem 3 .

Finally, by taking the functions $f_{i}(z)$ given by

$$
f_{i}(z)=a_{1, i} z-\frac{(A-B) \beta(1-\alpha) a_{1, i}}{2(1-\beta B)} z^{2} \quad(i=1,2)
$$

we can see that the result is sharp.

Corollary 3. For $f_{1}(z)$ and $f_{2}(z)$ as in Theorem 3, we have

$$
h(z)=\sqrt{a_{1,1} a_{1,2}} z-\sum_{n=2}^{\infty} \sqrt{a_{n, 1} a_{n, 2}} z^{n}
$$

belonges to the class $P^{*}(A, B, \alpha, \beta)$.

Proof. This result follows form the Cauchy-Schwarz inequality (4.7). It is sharp for the same functions as in Theorem 3 .

Theorem 4. Let the function $f_{1}(z)$ defined by (4.1) be in the class $P^{*}(A, B, \alpha, \beta)$ and the function $f_{2}(z)$ defined by (4.1) be in the class $P^{*}(A, B, \gamma, \beta)$, then $f_{1} * f_{2}(z) \in P^{*}(A, B, \zeta(A, B, \alpha, \gamma, \beta), \beta)$, where

$$
\zeta(A, B, \alpha, \gamma, \beta)=1-\frac{(A-B) \beta(1-\alpha)(1-\gamma)}{2(1-\beta B)} .
$$


The result is sharp.

Proof. Proceeding as in the proof of Theorem 3, we get

$$
\zeta \leq D(n)=1-\frac{(A-B) \beta(1-\alpha)(1-\gamma)}{n(1-\beta B)}(n \geq 2)
$$

Since the function $D(n)$ is an increasing function of $n(n \geq 2)$, letting $n=2$ in (4.18), we obtain

$$
\zeta \leq D(2)=1-\frac{(A-B) \beta(1-\alpha)(1-\gamma)}{2(1-\beta B)},
$$

which evidently proves Theorem 4 . Finally the result is best possible for the functions

$$
f_{1}(z)=a_{1,1} z-\frac{(A-B) \beta(1-\alpha) a_{1,1}}{2(1-\beta B)} z^{2}
$$

and

$$
f_{2}(z)=a_{1,2} z-\frac{(A-B) \beta(1-\gamma) a_{1,2}}{2(1-\beta B)} z_{2}
$$

Corollary 4. Let the functions $f_{i}(z)(i=1,2,3)$ defined by (4.1) be in the class $P^{*}(A, B, \alpha, \beta)$, then $f_{1} * f_{2} * f_{3}(z) \in P^{*}(A, B, \eta(A, B, \alpha, \beta), \beta)$, where

$$
\eta(A, B, \alpha, \beta)=1-\frac{(A-B)^{2} \beta^{2}(1-\alpha)^{3}}{4(1-\beta B)^{2}} .
$$

The result is best possible for the functions

$$
f_{i}(z)=a_{1, i} z-\frac{(A-B) \beta(1-\alpha) a_{1, i}}{2(1-\beta B)} z^{2} \quad(i=1,2,3) .
$$

Proof. From Theorem 3, we have $f_{i} * f_{2}(z) \in P^{*}(A, B, \delta(A, B, \alpha, \beta), \beta)$, where $\delta$ is given by (4.3). We now use Theorem 4 , we get $f_{1} * f_{2} * f_{3}(z) \in P^{*}(A, B, \eta(A, B, \alpha, \beta), \beta)$, where

$$
\eta(A, B, \alpha, \beta)=1-\frac{(A-B) \beta(1-\alpha)(1-\delta)}{2(1-\beta B)}=1-\frac{(A-B)^{2} \beta^{2}(1-\alpha)^{3}}{4(1-\beta B)^{2}} \text {. }
$$

This completes the proof of Corollary 4 .

\section{Fractional Calculus}

We begin with the statements of the following definitions of fractional calculus (that is, fractional derivatives and fractional integrals) which were defined by Owa [5]. 
Definition 2. The fractional integral of order $k(k>0)$ is defined, for a function $f(z)$, by

$$
D_{z}^{-k} f(z)=\frac{1}{\Gamma(k)} \int_{0}^{z} \frac{f(\zeta)}{(z-\zeta)^{1-k}} d \zeta
$$

where $f(z)$ is an analytic function in a simply-connected region of the $z$-plane containing the origin, and the multiplicity of $(z-\zeta)^{k-1}$ is removed by requiring $\log (z-\zeta)$ to be real when $z-\zeta>0$.

Definition 3. The fractional derivative of order $k(0 \leq k<1)$ is defined, for a function $f(z)$, by

$$
D_{z}^{k} f(z)=\frac{1}{\Gamma(1-k)} \frac{d}{d z} \int_{0}^{z} \frac{f(\zeta)}{(z-\zeta)^{k}} d \zeta
$$

where $f(z)$ is constrained, and the multiplicity of $(z-\zeta)^{-k}$ is removed, as in Definition 2 .

Definition 4. Under the hypotheses of Definition 3, the fractional derivative of order $n+k\left(0 \leq k<1 ; n \in \mathrm{N}_{0}=\{0,1, \ldots\}\right)$ is defined by

$$
D_{z}^{n+k} f(z)=\frac{d^{n}}{d z^{z}} D_{z}^{d} f(z)
$$

Theorem 5. Let the function $f(z)$ defined by (1.1) be in the class $P^{*}(A, B, \alpha, \beta)$. Then we have

$$
\left|D_{z}^{-k} f(z)\right| \geq \frac{a_{1}|z|^{1+k}}{\Gamma(2+k)}\left[1-\frac{(A-B) \beta(1-\alpha)}{(2+k)(1-\beta B)}|z|\right]
$$

and

$$
\left|D_{z}^{-k} f(z)\right| \leq \frac{a_{1}|z|^{1+k}}{\Gamma(2+k)}\left[1+\frac{(A-B) \beta(1-\alpha)}{(2+k)(1-\beta B)}|z|\right]
$$

for $k>0$ and $z \in U$. The result is sharp.

Proof. Let

$$
\begin{aligned}
F(z) & =\Gamma(2+k) z^{-k} D_{z}^{-k} f(z) \\
& =a_{1} z-\sum_{n=2}^{\infty} \frac{\Gamma(n+1) \Gamma(2+k)}{\Gamma(n+1+k)} a_{n} z^{n} \\
& =a_{1} z-\sum_{n=2}^{\infty} \psi(n) a_{n} z^{n}
\end{aligned}
$$

where

$$
\psi(n)=\frac{\Gamma(n+1) \Gamma(2+k)}{\Gamma(n+1+k)} \quad(n \geq 2) .
$$


Since

$$
0<\psi(n) \leq \psi(2)=\frac{2}{2+k}
$$

for $k>0$ and $n \geq 2$. Therefore, by using (3.5) and (5.8), we can see that

$$
\begin{aligned}
|F(z)| & \geq a_{1}|z|-\psi(2)|z|^{2} \sum_{n=2}^{\infty} a_{n} \\
& \geq a_{1}|z|-\frac{(A-B) \beta(1-\alpha) a_{1}}{(2+k)(1-\beta B)}|z|^{2}
\end{aligned}
$$

which implies (5.4), and

$$
\begin{aligned}
|F(z)| & \leq a_{1}|z|+\psi(2)|z|^{2} \sum_{n=2}^{\infty} a_{n} \\
& \leq a_{1}|z|+\frac{(A-B) \beta(1-\alpha) a_{1}}{(2+k)(1-\alpha B)}|z|^{2}
\end{aligned}
$$

which shows (5.5). Further, equalities are attained for the function

$$
D_{z}^{-k} f(z)=\frac{a_{1} z^{1+k}}{\Gamma(2+k)}\left[1-\frac{(A-B) \beta(1-\alpha)}{(2+k)(1-\beta B)} z\right]
$$

or for the function $f(z)$ given by (3.3). This completes the proof of Theorem 5 .

Corollary 5. Under the hypotheses of Theorem $5, D_{z}^{-k} f(z)(k>0, z \in U)$ is included in a disc with its center at the origin and the radius $r_{1}$ given by

$$
r_{1}=\frac{a_{1}}{\Gamma(2+k)}\left[1+\frac{(A-B) \beta(1-\alpha)}{(2+k)(1-\beta B)}\right] .
$$

Theorem 6. Let the function $f(z)$ defined by (1.1) be in the class $P^{*}(A, B, \alpha, \beta)$. Then we have

$$
\begin{aligned}
& \left|D_{z}^{k} f(z)\right| \geq \frac{a_{1}|z|^{1-k}}{\Gamma(2-k)}\left[1-\frac{(A-B) \beta(1-\alpha)}{(2-k)(1-\beta B)}|z|\right] \\
& \left|D_{z}^{k} f(z)\right| \leq \frac{a_{1}|z|^{1-k}}{\Gamma(2-k)}\left[1+\frac{(A-B) \beta(1-\alpha)}{(2-k)(1-\beta B)}|z|\right]
\end{aligned}
$$

for $0 \leq k<1$ and $z \in U$. The result is sharp.

Proof. Let

$$
\begin{aligned}
G(z) & =\Gamma(2-k) z^{k} D_{z}^{k} f(z) \\
& =a_{1} z-\sum_{n=2}^{\infty} \frac{\Gamma(n+1) \Gamma(2-k)}{\Gamma(n+1-k)} a_{n} z^{n} \\
& =a_{1} z-\sum_{n=2}^{\infty} \Phi(n) n a_{n} z^{n}
\end{aligned}
$$


where

$$
\Phi(n)=\frac{\Gamma(n) \Gamma(2-k)}{\Gamma(n+1-k)} \quad(n \geq 2)
$$

Noting that

$$
0<\Phi(n) \leq \Phi(2)=\frac{1}{2-k}
$$

for $0 \leq k<1$ and $n \geq 2$. Therefore, by using (3.8) and (5.17), we can see that

$$
\begin{aligned}
|G(z)| & \geq a_{1}|z|-\Phi(2)|z|^{2} \sum_{n=2}^{\infty} n a_{n} \\
& \geq a_{1}|z|-\frac{(A-B) \beta(1-\alpha) a_{1}}{(2-k)(1-\beta B)}|z|^{2}
\end{aligned}
$$

which implies (5.13), and

$$
\begin{aligned}
|G(z)| & \leq a_{1}|z|+\Phi(2)|z|^{2} \sum_{n=2}^{\infty} n a_{n} \\
& \leq a_{1}|z|+\frac{(A-B) \beta(1-\alpha) a_{1}}{(2-k)(1-\beta B)}|z|^{2}
\end{aligned}
$$

which implies (5.14). Further, equalities are attained by the function

$$
D_{z}^{k} f(z)=\frac{a_{1} z^{1-k}}{\Gamma(2-k)}\left[1-\frac{(A-B) \beta(1-\alpha)}{(2-k)(1-\beta B)} z\right]
$$

or by the function $f(z)$ given by (3.3). This completes the proof of Theorem 6 .

Corollary 6. Under the hypotheses of Theorem $6, d_{z}^{k} f(z)(0 \leq k<1, z \in U)$ is included in a disc with its center at the origin and the radius $r_{2}$ given by

$$
r_{2}=\frac{a_{1}}{\Gamma(2-k)}\left[1+\frac{(A-B) \beta(1-\alpha)}{(2-k)(1-\beta B)}\right]
$$

\section{Fractional Integral Operator}

We need the following definition of fractional integral operator given by Srivastava, Saigo and Owa [12].

Definition 5. For real numbers $\zeta>0, \gamma$ and $\tau$, the fractional operator $I_{0, z}^{\zeta, \gamma, \tau}$ is defined by

$$
I_{0, z}^{\zeta, \gamma, \tau} f(z)=\frac{z^{-\zeta-\gamma}}{\Gamma(\zeta)} \int_{0}^{z}(z-t)^{\zeta-1} F\left(\zeta+\gamma,-\tau ; \zeta ; 1-\frac{t}{z}\right) f(t) d t
$$


where $f(z)$ is an analytic function in a simply connected region of the $z$-plane containing the origin with the order

$$
f(z)=O\left(|z|^{\epsilon}\right), \quad z \longrightarrow 0
$$

where

$$
\begin{gathered}
\epsilon>\operatorname{Max}(0, \gamma-\tau)-1 \\
F(a, b ; c ; z)=\sum_{n=0}^{\infty} \frac{(a)_{n}(b)_{n}}{(c)_{n}(1)_{n}} z^{n}
\end{gathered}
$$

with $(\nu)_{n}$ being the Pochhammer symbol

$$
(\nu)_{n}=\frac{\Gamma(\nu+n)}{\Gamma(n)}=\left\{\begin{array}{ll}
1 & (n=0) \\
\nu(\nu+1) \ldots(\nu+n-1) & (n \in N=\{1,2, \ldots\})
\end{array} .\right.
$$

and the multiplicity of $(z-t)^{\zeta-1}$ is removed by requiring $\log (z-t)$ to be real when $z-t>0$.

Remark 2. For $\gamma=-\zeta$, we note that

$$
I_{0, z}^{\zeta,-\zeta, \tau} f(z)=D_{z}^{-\zeta} f(z)
$$

In order to prove our result for the fractional integral operator, we have to recall the following lemma due to Srivastava, Saigo and Owa [12].

Lemma 1. If $\zeta>0$ and $n>\gamma-\tau-1$, then

$$
I_{0, z}^{\zeta, \gamma, \tau} z^{n}=\frac{\Gamma(n+1) \Gamma(n-\gamma+\tau+1)}{\Gamma(n-\gamma+1) \Gamma(n+\zeta+\tau+1)} z^{n-\gamma}
$$

With the aid of Lemma 1, we have

Theorem 7. Let $\zeta>0, \gamma<2, \gamma+\tau>-2, \gamma-\tau<2, \gamma(\zeta+\tau) \leq 3 \zeta$. If the function $f(z)$ defined by (1.1) is in the class $P^{*}(A, B, \alpha, \beta)$, then

$$
\begin{aligned}
\left|I_{0, z}^{\zeta, \gamma, \tau} f(z)\right| \geq & \frac{a_{1} \Gamma(2-\gamma+\tau)|z|^{1-\gamma}}{\Gamma(2-\gamma) \Gamma(2+\zeta+\tau)} \\
& \cdot\left\{1-\frac{(A-B) \beta(1-\alpha)(2-\gamma+\tau)}{(1-\beta B)(2-\gamma)(2+\zeta+\tau)}|z|\right\}
\end{aligned}
$$

and

$$
\begin{aligned}
\left|I_{0, z}^{\zeta, \gamma, \tau} f(z)\right| \leq & \frac{a_{1} \Gamma(2-\gamma+\tau)|z|^{1-\gamma}}{\Gamma(2-\gamma) \Gamma(2+\zeta+\tau)} \\
& \cdot\left\{1+\frac{(A-B) \beta(1-\alpha)(2-\gamma+\tau)}{(1-\beta B)(2-\gamma)(2+\zeta+\tau)}|z|\right\}
\end{aligned}
$$


for $z \in U_{O}$, where

$$
U_{O}= \begin{cases}U & (\gamma \leq 1) \\ U-\{O\} & (\gamma>1) .\end{cases}
$$

The equalities in (6.5) and (6.6) are attained by the function $f(z)$ given by (3.3).

Proof. By using Lemma 1, we have

$$
I_{0, z}^{\zeta, \gamma, \tau} f(z)=\frac{a_{1} \Gamma(2-\gamma+\tau)}{\Gamma(2-\gamma) \Gamma(2+\zeta+\tau)} z^{1-\gamma}-\sum_{n=2}^{\infty} \frac{\Gamma(n+1) \Gamma(n-\gamma+\tau+1)}{\Gamma(n-\gamma+1) \Gamma(n+\zeta+\tau+1)} a_{n} z^{n-\gamma}
$$

Letting

$$
\begin{aligned}
H(z) & =\frac{\Gamma(2-\gamma) \Gamma(2+\zeta+\tau)}{\Gamma(2-\gamma+\tau)} z^{\gamma} I_{0, z}^{\zeta, \gamma, \tau} f(z) \\
& =a_{1} z-\sum_{n=2}^{\infty} h(n) a_{n} z^{n}
\end{aligned}
$$

where

$$
h(n)=\frac{(2-\gamma+\tau)_{n-1}(1)_{n}}{(2-\gamma)_{n-1}(2+\zeta+\tau)_{n-1}} \quad(n \geq 2),
$$

we can see that $h(n)$ is non-increasing for integers $n \geq 2$, and we have

$$
0<h(n) \leq h(2)=\frac{2(2-\gamma+\tau)}{(2-\gamma)(2+\zeta+\tau)} .
$$

Therefore, by using (3.5) and (6.10), we have

$$
\begin{aligned}
|H(z)| & \geq a_{1}|z|-h(2)|z|^{2} \sum_{n=2}^{\infty} a_{n} \\
& \geq a_{1}|z|-\frac{(A-B) \beta(1-\alpha)(2-\gamma+\tau) a_{1}}{(1-\beta B)(2-\gamma)(2+\zeta+\tau)}|z|^{2}
\end{aligned}
$$

and

$$
\begin{aligned}
|H(z)| & \leq a_{1}|z|-h(2)|z|^{2} \sum_{n=2}^{\infty} a_{n} \\
& \leq a_{1}|z|-\frac{(A-B) \beta(1-\alpha)(2-\gamma+\tau) a_{1}}{(1-\beta B)(2-\gamma)(2+\zeta+\tau)}|z|^{2} .
\end{aligned}
$$

This complets the proof of Theorem 7 .

Remark 3. Taking $\gamma=-\zeta=-k$ in Theorem 7 , we get the result of Theorem 5 .

Remark 4. Owa [7] considered the class $P_{0}^{*}(\alpha, \beta)$ of functions $f(z)=a_{1} z-$ $\sum_{n=2}^{\infty} a_{n} z^{n}\left(a_{n} \geq 0 ; a_{1}>0\right)$ analytic and univelent in $U$ and satisfying

$$
\left|\frac{f^{\prime}(z)-1}{f^{\prime}(z)+(1-2 \alpha)}\right|<\beta, z \in U
$$


where $0 \leq \alpha<1$ and $0<\beta \leq 1$.

One can easily verify that the condition (i) is equivelent to

$$
f^{\prime}(z)=\frac{1+\beta(1-2 \alpha) \omega(z)}{1-\beta \omega(z)}, z \in U
$$

where $\omega(z)$ is a function analtyic in $U$ and satisfying $\omega(0)=0$ and $|\omega(z)|<1$ for $z \in U$. Since $f^{\prime}(z)=a_{1}-\sum_{n=2}^{\infty} n a_{n} z^{n-1}$, it follows that the constant term in the Taylor expansion of both sides of (ii) is not the same except when $a_{1}=1$. It seems, therefore, that the class $P_{0}^{*}(\alpha, \beta)$ has not been defined by Owa [7] in proper way. in fact, the correct form of (i) must be

$$
\left|\frac{f^{\prime}(z)-a_{1}}{f^{\prime}(z)+(1-2 \alpha) a_{1}}\right|<\beta, z \in U
$$

where we put $A=-1 B=1$ in (1.3). Consequently, the correct form of (ii) is

$$
f^{\prime}(z)=a_{1} \frac{1+\beta(1-2 \alpha) \omega(z)}{1-\beta \omega(z)}, z \in U
$$

Acknowledgements. The auther would like to thank the referee of the paper for his helpful suggestions.

\section{References}

[1] M. K. Aouf, "On certain classes of univalent function, with negative coefficients" (Submitted)

[2] V. P. Gupta and I. Ahamed, "Certain classes of univalent functions in the unit disc," Bull. Inst. Math. Acad. Sinica, 5(1977),379-389.

[3] V. P. Gupta and P. K. Jain, "Certain classes of univalent functions with negative coefficients," Bull. Austral. Math. Soc. 14(1976), 409-416.

[4] V. P. Gupta and P. K. Jain, "Certain classes of univalent functions with negative coefficients. II," Bull. Austral. Math. Soc. 15(1976), 467-473.

[5] S. Owa, "On the distortion theorems. I," Kyungpook Math. J. 18(1978), 53-59.

[6] S. Owa, "On the classes of univalent functions with negative coefficients." Math. Japon. 27(1982), 409-416.

[7] S.Owa, "On the special classes of univalent functions," Tamkang J. Math. 15(1984), no. 2, 123-136.

[8] S. Owa, "A remark on the special classes of univalent functions," Math. Japon. 27(1982), no.5, 625-630.

[9] S. Owa and M. K. Aouf, "On subclasses of univalent functions with negative cofficients. II," Pure Appl. Math. Sci. 29(1989), no. 1-2, 131-139.

[10] T. Sekine, S Owa and K. Nishimoto, "An application of the fractional calculus," J. Colllege Engng. Nihion Univ., Ser. B, 27(1986), 31-37.

[11] A. Schild and H. Silverman, "Convolitions of univalent functions with negative coefficients," Ann. Univ. Mariae Curie-Sklodowska Sect. A, 29(1975), 99-107.

[12] H. M. Srivastava, M. Saigo and S. Owa, "A class of distortion theorems involving certain operators of fractional calculs," J. Math. Anal. Appl. 131(1988), 412-420.

Department of Mathematics, Faculty of science, University of Mansoura, Mansoura, Egypt. 